\title{
RESCUING THE INTRACLUSTER MEDIUM OF NGC 5813
}

\author{
Noam Soker ${ }^{1}$, Shlomi Hillel ${ }^{1}$, and Assaf Sternberg ${ }^{2}$
}

\begin{abstract}
We use recent X-ray observations of the intracluster medium (ICM) of the galaxy group NGC 5813 to confront theoretical studies of ICM thermal evolution with the newly derived ICM properties. We argue that the ICM of the cooling flow galaxy group NGC 5813 is more likely to be heated by mixing of post-shock jets' gas residing in hot bubbles with the ICM, than by shocks or turbulent-heating. Shocks thermalize only a small fraction of their energy in the inner regions of the cooling flow; in order to adequately heat the inner part of the ICM, they would overheat the outer regions by a large factor, leading to its ejection from the group. Heating by mixing, that was found to be much more efficient than turbulent-heating and shocks-heating, hence, rescues the outer ICM of NGC 5813 from its predestined fate according to cooling flow feedback scenarios that are based on heating by shocks.
\end{abstract}

Keywords: galaxies: active galaxies: clusters: general galaxies: groups: individual (NGC 5813) - galaxies: jets

\section{INTRODUCTION}

A negative feedback mechanism driven by active galactic nucleus (AGN) jets that inflate Xray bubbles (X-ray deficient cavities) determines the thermal evolution of the intra-cluster medium (ICM) in the inner regions of cooling flow (CF) clusters and groups of galaxies (e.g., Pfrommer 2013; Perucho et al. 2014). In the feedback cycle the ICM feeds the AGN, and the AGN launches jets that heat the ICM.

A consensus is emerging that the feeding of the AGN is through cold clumps, in what is termed the cold feedback mechanism (Pizzolato \& Soker 2005). Observations and theoretical studies in recent years have put the cold feedback mechanism on a very solid ground (e.g., Revaz et al. 2008; Pope 2009; Pizzolato \& Soker 2010; Edge et al. 2010; Wilman et al. 2011; Nesvadba et al. 2011; Cavagnolo et al.|2011; Gaspari et al. 2012a. b; |McCourt et al. 2012; Sharma et al.|2012; Farage et al. 2012; Wagh et al. 2014; Banerjee \& Sharma 2014; McNamara et al. 2014; Voit \& Donahue 2015;

\footnotetext{
${ }^{1}$ Department of Physics, Technion - Israel Institute of Technology, Haifa 32000, Israel; soker@physics.technion.ac.il; shlomihi@tx.technion.ac.il

${ }^{2}$ Geneva Observatory, University of Geneva, Chemin des Maillettes 51, CH-1290 Versoix, Switzerland; assaf.sternberg@unige.ch
} 
Voit et al. 2015; Li et al. 2015; Prasad et al. 2015; Russell et al. 2015; Tremblay et al. 2015; Fogarty et al. 2015).

The processes by which jets heat the ICM, on the other hand, is still in dispute. Based on observations of the galaxy group NGC 5813 Randall et al. (2011) and Randall et al. (2015) argue that shocks heat the ICM; hereafter shocks-heating process. This was put into question by Soker et al. (2013), and is further studied in the present paper. Based on deep X-ray observation of the Perseus and Virgo cooling flow clusters, Zhuravleva et al. (2014) argue that the main heating process is via dissipation of ICM turbulence; hereafter turbulent-heating process. The third heating process is the mixing of cooling ICM with hot shocked jets' gas that reside in the hot bubbles; hereafter mixingheating process. Gilkis \& Soker (2012) and Hillel \& Soker (2014) conducted numerical simulations and showed that the mixing-heating process is much more efficient than the shocks-heating process. In a recent paper Hillel \& Soker (2016) showed that the mixing-heating is also more efficient than the turbulent-heating when turbulence is driven by AGN activity. The turbulence, as observed in some clusters (e.g., Zhuravleva et al. 2015; Anderson \& Sunvaev 2015), is a consequence of the same vortices that cause mixing.

To reach the conclusion that mixing-heating is the dominant process, it is mandatory to inflate bubbles by jets in a self-consistent manner. For example, the vortices that are induced inside and outside the bubbles must be taken into account as they play major roles in the formation of bubbles, their evolution, and their interaction with the ICM, (e.g. Omma et al. 2004; Heinz \& Churazov 2005; Roediger et al. 2007; Sternberg \& Soker 2008b; Gilkis \& Soker 2012; Walg et al. 2013). To inflate bubbles that resemble observed bubbles, our group has employed either slow (sub-relativistic) massive wide (SMW) jets (bipolar outflows), e.g., (Sternberg et al. 2007), or precessing jets, e.g., (Sternberg \& Soker 2008a); see also Falceta-Goncalves et al. (2010). SMW jets are supported by observations (e.g., Moe et al. 2009; Dunn et al. 2010; Arav et al. 2013). Both types of inflated bubbles will be described in the present study.

In a recent paper Randall et al. (2015) presented new observations of NGC 5813. Such detailed observations serve as a unique opportunity for us to confront our findings, regarding heating the ICM with jet-inflated bubbles, against observations. We here confront the new observations with two types of previously conducted numerical simulations. In section 3 we present a new study of the implications on the heating process of large scale flows that are induced by the jet-inflated bubbles. In section 4 we show that a careful treatment is required when studying the shocks that are induced by jet-inflated bubbles. To our best knowledge, this is the first study that point to the influence of sound waves on the derived shock properties. But we first present in section 2 a new analysis of the effect on many repeated shocks on the outskirts of the ICM in NGC 5813. In section 5 we summarize our findings, and conclude that although shocks play some role, mixing-heating is likely to play a much more significant role in the heating process. 


\section{IMPLICATIONS FOR THE OUTER ICM}

The inefficiency of the shocks-heating process was discussed in Soker et al. (2013). To excite a shock with a Mach number of $\mathcal{M}_{\mathrm{s}}$ at radius $r$, the energy supplied by the AGN directly to the ICM, using the spherically symmetric Sedov solution for the shock, should be

$$
E_{\text {shock }} \approx\left(\frac{\mathcal{M}_{\mathrm{s}}}{1.1}\right)^{2} E_{\mathrm{th}}(r)
$$

where $E_{\mathrm{th}}(r)$ is the thermal energy of the ICM inside radius $r$. According to Randall et al. (2015), to heat the cooling flow region of NGC 5813 inside $30 \mathrm{kpc}$, about 140 shocks with a Mach number of $\mathcal{M}_{a} \approx 1.2$ are required during one cooling time. By equation (1), to reach this Mach number each shock is excited with an energy of $E_{\text {shock }} \approx(1.2 / 1.1)^{2} E_{\text {in }}=1.2 E_{\text {in }}$, where we define $E_{\text {in }} \equiv$ $E_{\mathrm{th}}(r<30 \mathrm{kpc})$. Each shock supplies an energy of $\approx E_{\mathrm{in}} / 140=0.01 E_{\mathrm{in}}$. Removing this dissipated energy in the inner region (up to $30 \mathrm{kpc}$ ), the energy that flows to the outer ICM regions within one cooling time at $r=30 \mathrm{kpc}$ is $E_{\text {out }} \approx 140(1.2-0.01) E_{\text {in }} \approx 160 E_{\text {in }}$.

The ICM mass residing out at $r>30 \mathrm{kpc}$ can be crudely estimated from the new density profile given by Randall et al. (2015). The electron density profile from $r=22 \mathrm{kpc}$ to $r=35 \mathrm{kpc}$, the largest radius in their profile, can be fitted with

$$
n_{e}(r)=2.4 \times 10^{-3}\left(\frac{r}{30 \mathrm{kpc}}\right)^{-2.8} \mathrm{~cm}^{-3} .
$$

Extrapolating this fit to large distances of hundreds of kpc, we can estimate the mass in the outer region to be $M_{\text {out }} \approx 10^{11} M_{\odot} \approx 2 M_{\text {in }}$. Based on ROSAT observations of poor groups of galaxies Mulchaev et al. (1996) find the diffuse X-ray gas in a typical poor group to extend up to $\sim 300 \mathrm{kpc}$, and have a shallower density profile. If we take a shallower density profile of

$$
n_{e-s}(r)=2.4 \times 10^{-3}\left(\frac{r}{30 \mathrm{kpc}}\right)^{-1.5} \mathrm{~cm}^{-3},
$$

(like in eq. 5 below), the gas mass in the outer region to a distance of $300 \mathrm{kpc}$ is $M_{\text {out-s }} \approx$ $5 \times 10^{11} M_{\odot} \approx 10 M_{\text {in }}$

Randall et al. (2015) do mention that the energy carried by the bubble is transferred out by the buoyant bubbles. But the shocks turn into sound wave that dissipate their energy after a long time in the ICM. By the simple estimate made above the temperature of the outer gas should be

$$
T_{\text {out }} \approx 10\left(\frac{T_{\text {in }}}{0.7 \mathrm{keV}}\right)\left(\frac{E_{\text {out }}}{150 E_{\text {in }}}\right)\left(\frac{M_{\text {out }}}{10 M_{\text {in }}}\right)^{-1} \mathrm{keV} .
$$

This is a temperature larger than the virial temperature of the group.

It seems that for the shocks-heating process to be effective in the inner region, the outer ICM would escape in a time much shorter than the cooling time, hence destroying the ICM observed structure. Clearly, a much more efficient heating mechanism than shocks is required to rescue the ICM of NGC 5813. Mixing-heating is this process (Hillel \& Soker 2014, 2016). 


\section{LARGE SCALE FLOW}

In the shocks-heating scenario presented by Randall et al. (2011) and Randall et al. (2015) tens of jet-launching episodes along the same axis heat the ICM. They do not consider any flow that might develop in the ICM. We here show that a large-scale meridional flow develops as a result of multi-jet activity cycles. For that we present results from 3D numerical simulations we have carried out recently (Hillel \& Soker 2016). We here briefly present the main characteristics of the numerical set up; more details can be found in that paper.

We use the PLUTo code (Mignone et al. 2007) for the hydrodynamic simulations in a threedimensional Cartesian grid with adaptive mesh refinement (AMR). The computational grid is in the octant where the three coordinates $x, y$ and $z$ are positive. At the $x=0, y=0$ and $z=0$ planes we apply reflective boundary conditions. The $z$ coordinate is chosen along the initial axis of the jets. The computational grid spans the cube $0 \leq x, y, z \leq 50 \mathrm{kpc}$.

At the boundary $z=0$ we inject into the grid a jet with a half-opening angle of $\theta_{\mathrm{j}}=70^{\circ}$ (Sternberg et al. 2007). The jet is injected during each active episode lasting $10 \mathrm{Myr}$, and when the jet is turned off for $10 \mathrm{Myr}$, reflective boundary conditions apply in the whole $z=0$ plane. The initial jet velocity is $v_{\mathrm{j}}=8200 \mathrm{~km} \mathrm{~s}^{-1}$ corresponding to a Mach number of about 10 relative to the ICM. The power of the two jets together (we simulate only one jet) is $\dot{E}_{2 \mathrm{j}}=2 \times 10^{45} \mathrm{erg} \mathrm{s}^{-1}$ (half of it in each direction), and the mass deposition rate is $\dot{M}_{2 \mathrm{j}}=94 M_{\odot} \mathrm{yr}^{-1}$.

The simulations presented here assume jets starting with wide opening angles. As stated in section 1, there are observations of slow massive wide outflows from AGNs. As well, the effect of rapidly precessing jets in inflating bubbles is very similar to that of wide jets (Sternberg \& Soker 2008a), but it is simpler to simulate wide jets (for precessing jets see next section). The main issue is to inflate bubbles, a process that involves the formation of vortices. Although magnetic fields are present in jets, as inferred from the radio emission, we here assume that they do not play a dynamical role. As we heat by mixing, we attribute no role to heat conduction on large scales (only on very small scales as mixing brings the ICM and the hot bubble gas very close to each other).

The simulation begins with an isothermal box of gas at an initial temperature of $T_{\mathrm{ICM}}(0)=$ $3 \times 10^{7} \mathrm{~K}$ with a density profile of (e.g., Vernaleo \& Reynolds 2006)

$$
\rho_{\mathrm{ICM}}(r)=\frac{\rho_{0}}{\left[1+(r / a)^{2}\right]^{3 / 4}},
$$

with $a=100 \mathrm{kpc}$ and $\rho_{0}=10^{-25} \mathrm{~g} \mathrm{~cm}^{-3}$. A gravity field is added to maintain an initial hydrostatic equilibrium, and is kept constant in time. We include radiative cooling in the simulations, where the tabulated cooling function is taken from Table 6 in Sutherland \& Dopita (1993).

To reveal the large scale flow we follow an artificial flow quantity called 'tracer.' The tracer is frozen-in to the flow, and hence tells us on the spreading with time of gas starting in a certain volume. A tracer's initial value is set to $\xi(0)=1$ in a certain volume and $\xi(0)=0$ elsewhere. As the 
traced gas mixes with the ICM or the jet's material, its value drops to $0<\xi(t)<1$. We choose to trace the gas starting inside a torus around the $z$ axis. The radius of the cross section of the torus is $2.5 \mathrm{kpc}$, and it is centered at $(y, z)=(20,15) \mathrm{kpc}$; or more generally $\left(\sqrt{x^{2}+y^{2}}, z\right)=(20,15) \mathrm{kpc}$.

In Fig. 1 we present the evolution of the tracer at six times in the meridional plane $(y, z)$. Note that the jets' axis is along the $z$ axis, which is the horizontal axis in the figure. The evolution of other quantities in the $(x, z)$ meridional plane is presented by Hillel \& Soker (2016). In the panels on the left column the arrows represent flow velocity, with length linear with velocity up to an upper limit of $v_{m}=400 \mathrm{~km} \mathrm{~s}^{-1}$. Faster regions are presented with an arrow length corresponding to $v_{m}$. In the panels on the right column the arrows represent mass flux $\phi=v \rho$.

There are several prominent properties of the flow that are relevant to the present study and are revealed in Fig. 1.

1. Mixing. The vortices created during the inflation of the bubble mix hot bubble gas with the ICM gas. This can be clearly seen by following the evolution of the tracer. This is the main heating mechanism of the ICM by jets (Gilkis \& Soker 2012; Hillel \& Soker 2014, 2016).

2. Outward then inward motion. The shocks running through the ICM followed by sound waves behind them can push gas out. The traced gas presented here is pushed from a torus cross section centered at $(y, z)=(20,15) \mathrm{kpc}$ to an elongated shape centered at $(y, z) \simeq(29,15) \mathrm{kpc}$ at $t \simeq 100$ Myr. However, then it turns around and flows inward. It is heated only when it starts to be mixed with the hot bubble gas at $t \simeq 110$ Myr (Hillel \& Soker 2016).

3. Large scale meridional flow. At late times of $t \gtrsim 200 \mathrm{Myr}$, and after about 10 jet-activity episodes (cycles), a large-scale inflow takes place near the equatorial plane. This results from the dragging of ICM gas by the outflowing jets along their axis, here the horizontal $z$-axis. It seems though that in reality the jets' axis will change direction and no such large meridional flow will be develop. This change in direction can be seen in the three bubble-pairs in NGC 5813 (Randall et al. 2015).

It should be noted that the simulations presented here are general, and have parameters that fit clusters of galaxies, and do not fit groups of galaxies, such as NGC 5813. For example, the power of jets here is 200-1000 times the power inferred for the bubble formation in NGC 5813 (Randall et al. 2015). The consequence is that the mixing region extends much beyond a radius of $30 \mathrm{kpc}$. For a much lower power of the jets as appropriate for NGC 5813, we would get a much smaller mixing region, one that fits the cooling flow region of NGC 5813.

The main conclusion of this section is that one cannot assume that jets activity repeats itself along the same direction for many episodes, while the ICM in the equatorial plane (the plane perpendicular to the jets' axis) does not flow inward. The flow inward will mix ICM gas with bubble gas, leading to heating by mixing that is much more efficient than the shocks-heating process. 

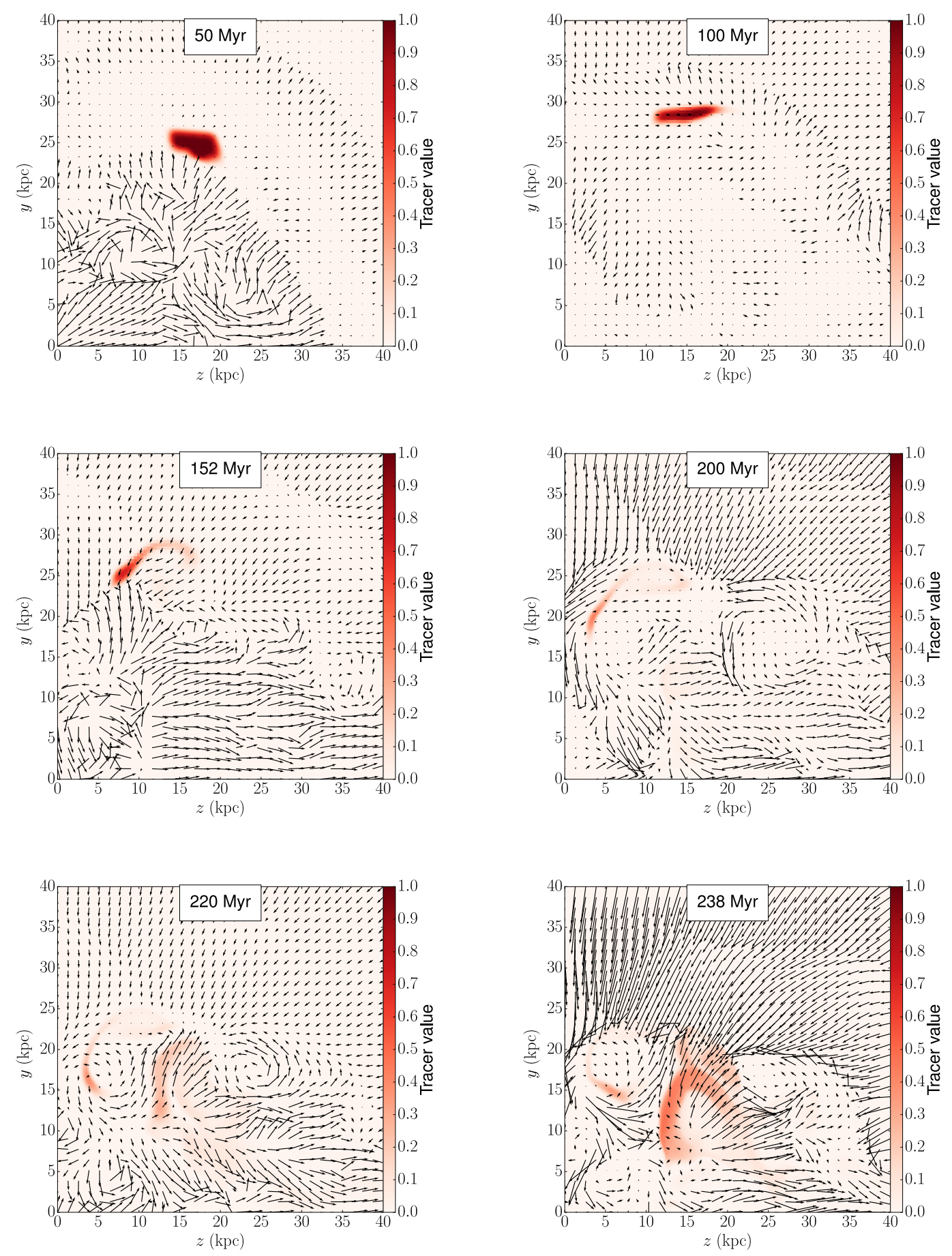

Fig. 1. - Evolution with time of a traced gas simulated by Hillel \& Soker (2016). At $t=0$ the traced gas was contained in a torus whose axis is the $z$-axis and whose cross section is a circle centered at $\left(\sqrt{x^{2}+y^{2}}, z\right)=(20,15) \mathrm{kpc}$ with a radius of $r=2.5 \mathrm{kpc}$. Color coding is of the tracer value $\xi$. The jets' axis is along the horizontal $z$-axis in the panels. In the three panels on the left the arrow lengths are linear with velocity, up to a maximum value of $v_{m}=400 \mathrm{~km} \mathrm{~s}^{-1}$. Faster regions have an arrow length corresponding to $v_{m}$. The arrows in the right panels show the mass flux $\phi=v \rho$. A length of $1 \mathrm{kpc}$ on the map corresponds to $\phi=2.3 \times 10^{-23} \mathrm{~km} \mathrm{~s}^{-1} \mathrm{~g} \mathrm{~cm}^{-3}$. 


\section{POST-SHOCK FLOW}

Randall et al. (2015) find the observed temperature rise across the shocks to be less than what is expected based on the Mach numbers they derive from their calculated density jumps across the shocks. They attribute the discrepancy to inability of the deprojected temperature measurements to resolve the temperature jump due to the narrow width of the shock and the rarefied cool gas behind the shock (Randall et al. 2011).

We here raise another possibility. When a bubble is inflated by a jet sound waves are excited and trail the shock wave (Sternberg \& Soker 2009). The sound waves can compress gas behind the shock, and raise the density behind the shock much more than they raise the temperature. In Figs. 2 and 3 we present results from Sternberg \& Soker (2009), where all technical details are given.

The relevant properties of these simulations are as follows. The simulation are performed in half of the meridional plane using the two-dimensional version of the VH1 code (Blondin et al. 1990; Stevens et al. 1992) in spherical coordinates with a resolution of $256 \times 256$. Gravity was included in these simulations. Two opposite jets are launched along the $x$-axis.

In the wide jets case two opposite jets were injected in the time period from $t=-10 \mathrm{Myr}$ until $t=0$, each jet with a half opening angle of $\alpha=70^{\circ}$. Their combined mass outflow rate was $\dot{M}_{2 \mathrm{j}}=10 M_{\odot} \mathrm{yr}^{-1}$ and the initial velocity was radial with $v_{j}=7750 \mathrm{~km} \mathrm{~s}^{-1}$. The total power of the two jets was $\dot{E}_{2 \mathrm{j}}=2 \times 10^{44} \mathrm{erg} \mathrm{s}^{-1}$. This case is presented in Fig. 2.

In the case of the precessing jets the two opposite jets have the same mass outflow rate and velocity as in the wide-jets case, but they were injected at any given time with a half opening angle of $\alpha=5^{\circ}$. In the axisymmetric grid employed, the jets are actually precessing very rapidly around the symmetry axis (i.e., in 3D torii were actually injected). The angle between the symmetry axis and the jets' axis $\theta$ was varied in a random way. The precession period, i.e., the time the jet returns to the same angle $\theta$, is $T_{\text {prec }}=0.1$ Myr. The jet's interaction with the ICM is similar to that of a wide jet with a half opening angle of $\alpha \approx 50^{\circ}$. The jets were active between $t=-18 \mathrm{Myr}$ and $t=0$. This case is presented in Fig. 3

The profiles along a cut in each case presented above show a density and temperature jump immediately behind the shock, the right edge in each of the two panels of profiles. Immediately behind the post-shock jump (moving to the left in the panels) there is another increase in the density, but the temperature decreases. The over all density jump overestimates the shock Mach number.

We argue that Randall et al. (2015) somewhat overestimate the Mach numbers in the shocks they study in NGC 5813. 

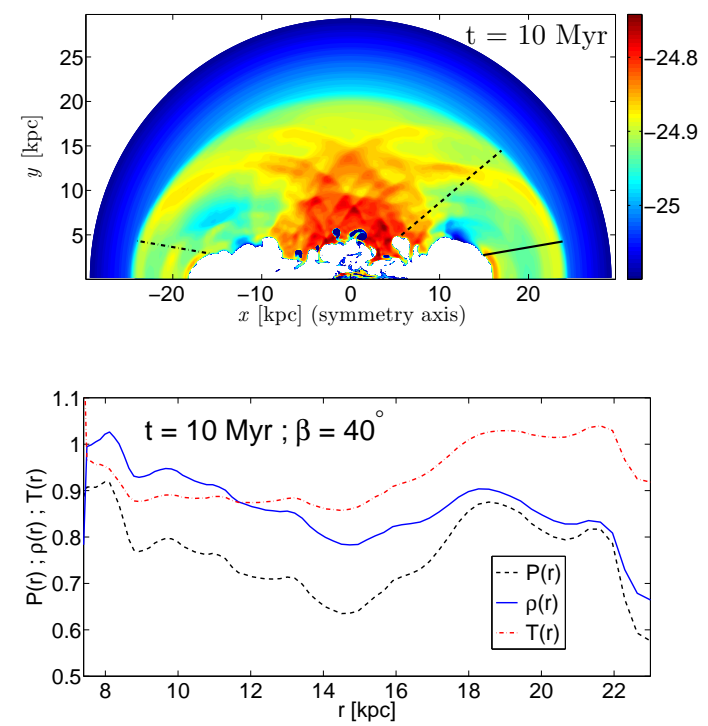

Fig. 2.- Excitation of sound waves by wide-jet-inflated bubbles. Upper panel: Density map for the ICM around the wide-jet-inflated bubbles at $t=10 \mathrm{Myr}$ after the jets has ceased. The density units of the color-code bar are $\log \rho\left(\mathrm{g} \mathrm{cm}^{-3}\right)$. The white regions are the two very-low density bubbles inflated by the two opposite jets. Lower panel: Density, temperature and pressure along the cut at $40^{\circ}$ as marked on the upper panel by the dashed line. The pressure is given in units of $6 \times 10^{-10} \mathrm{erg} \mathrm{cm}^{-3}$, density in units of $1.5 \times 10^{-25} \mathrm{~g} \mathrm{~cm}^{-3}$, and temperature in units of $2.7 \times 10^{7} \mathrm{~K}$. Both panels are taken from Sternberg \& Soker (2009) where all details are given. The post-shock region at $r=21.5 \mathrm{kpc}$ shows jumps in density, temperature, and pressure. The sound wave just behind the shock at $r=18.5 \mathrm{kpc}$ has density and pressure above the post-shock values, while the temperature is lower than the post-shock value. 

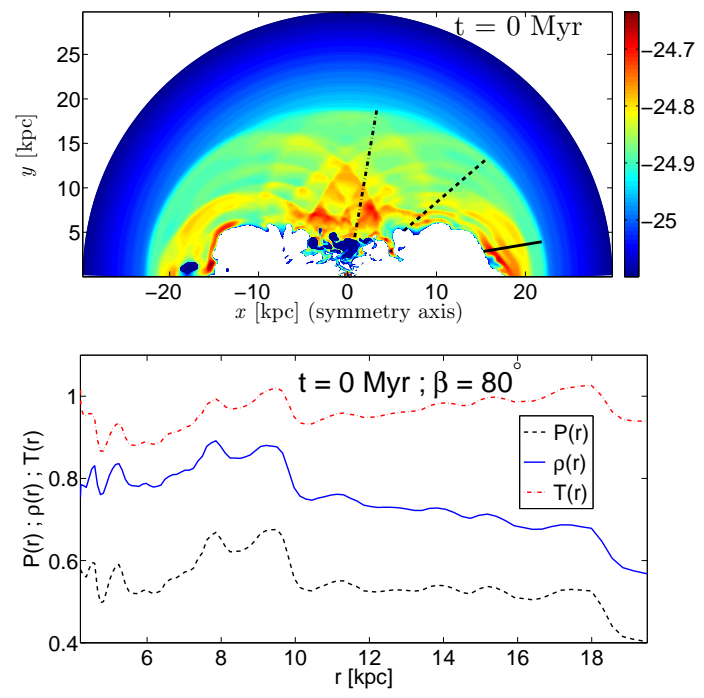

Fig. 3.- Excitation of sound waves by precessing-jet-inflated bubbles as simulated by Sternberg \& Soker (2009). Upper panel: Density map for the ICM around the precessing-jet-inflated bubbles at $t=0$, just when the jets cease. Lower panel: Density, temperature and pressure along the cut at $80^{\circ}$ as marked on the upper panel by the dashed-dotted line. The pressure is given in units of $9 \times 10^{-9} \mathrm{erg} \mathrm{cm}{ }^{-3}$, density in units of $2 \times 10^{-25} \mathrm{~g} \mathrm{~cm}^{-3}$, and temperature in units of $2.7 \times 10^{7} \mathrm{~K}$. The post-shock region at $r=18 \mathrm{kpc}$ shows jumps in density, temperature, and pressure. The sound wave behind the shock at $r=15.5 \mathrm{kpc}$ has density and pressure above the post-shock values, while the temperature is lower than the post-shock value. 


\section{SUMMARY}

We rexamined the claim made by Randall et al. (2011) and Randall et al. (2015) that the ICM in the cooling flow group of galaxies NGC 5813 is heated by many shock waves excited by repeated jet-launching episodes. It was already argued by Soker et al. (2013) that the shocksheating mechanism is inefficient, and cannot account for the NGC 5813 ICM properties. We here, however, performed three new analyses and confronted theoretical studies with the newly derived properties of the ICM of NGC 5813. ( $i$ ) We presented a new analytical analysis of the effect of many shocks on the outskirts of the ICM in NGC 5812. (ii) We discussed the role of the large scale flow induced by jet-inflated bubbles on the ICM and its thermal evolution. (iii) We used simulations of sound waves trailing shocks induced by jet-inflated bubbles to deduce about possible complications in deriving the shocks' properties from observations. These new analyses strengthen the conclusion that mixing-heating is more efficient that shock heating.

The energy carried by the shocks and that is not deposited in the inner region, $r \lesssim 30 \mathrm{kpc}$, is carried to the outer ICM regions and must dissipate there as sound waves. In section 2 we found that for the inefficient shocks-heating mechanism to heat the inner $r \lesssim 30 \mathrm{kpc}$ region, this extra energy heats the outer ICM to high enough temperature for it to escape the group of galaxies. If this was the case, the inner region would have expanded and escaped a long time ago. Clearly a more efficient heating mechanism is required.

In section 3 we showed that after about ten jet-launching episodes along a constant axis a large-scale meridional flow develops (Fig,1). An inflow of mass near the equatorial plane takes place. One cannot regard the ICM for such a case as static. Such a flow leads to a very vigorous mixing of the ICM with the hot bubbles' gas. Heating by mixing becomes the dominate heating mechanism even for gas perpendicular to the jets' axis (Gilkis \& Soker 2012; Hillel \& Soker 2014, 2016). We expect that in reality the jets' axis will change over time, and the meridional flow will not be as prominent as found here

In section 4 we showed that sound waves propagating behind a shock wave can form a region behind the shock where the density increases above the post-shock value, but the temperature does not. This might explain the finding of Randall et al. (2015) that the temperature jumps across the shocks in NGC 5813 are lower than those expected for the Mach numbers they deduced based on the density jumps. If true, it implies that Randall et al. (2015) overestimated the Mach numbers in the three shocks they have found in NGC 5813.

Our main conclusion is that shocks cannot be the main heating process of the ICM in NGC 5813. Heating by mixing seems to be the main heating process of the ICM by jets (Gilkis \& Soker 2012; Hillel \& Soker 2014, 2016). The mixing is caused by vortices that are formed during the inflation of the bubbles. To reveal the properties of the vortices in numerical simulations it is crucial to inflate bubbles in a self-consistent manner. When that is done, it is found that the mixing operates also perpendicular to the jets' axis. 
The vortices lead to turbulence in the ICM. Therefore, the finding of moderate turbulence in the inner region of NGC 5813 by Werner et al. (2009) and de Plaa et al. (2012), as well as in other clusters (Zhuravleva et al. 2014, 2015; Anderson \& Sunvaev 2015), strengthens the argument that the mixing-heating process is the dominate heating process. Some heating by the dissipation of turbulence takes place as well, but it accounts for at most $\approx 20 \%$ of the total heating (Hillel \& Soker 2016).

\section{Acknowledgements}

We thank an anonymous referee for helpful suggestions.

\section{REFERENCES}

Anderson, M., E., \& Sunyaev, R. 2015, arXiv:1506.01703

Arav, N., Borguet, B., Chamberlain, C., Edmonds, D., \& Danforth, C. 2013, MNRAS, 436, 3286

Banerjee, N. \& Sharma, P. 2014, MNRAS, 443, 687

Blondin, J. M., Kallman, T. R., Fryxell, B. A., \& Taam, R. E. 1990, ApJ, 356, 591

Cavagnolo, K. W., McNamara, B. R., Wise, M. W., Nulsen, P. E. J., Brüggen, M., Gitti, M., \& Rafferty, D. A. 2011, ApJ, 732, 71

de Plaa, J., Zhuravleva, I., Werner, N., Kaastra, J. S., Churazov, E., Smith, R. K., Raassen, A. J. J., \& Grange, Y. G. 2012, A\&A, 539, AA34

Dunn, J. P., Bautista, M., Arav, N., et al. 2010, ApJ, 709, 611

Edge, A. C., et al. 2010, A\&A, 518, L47

Falceta-Goncalves, D., Caproni, A., Abraham, Z., Teixeira, D. M., \& de Gouveia Dal Pino, E. M. 2010, ApJ, 713, L74

Farage, C. L., McGregor, P. J., \& Dopita, M. A. 2012, ApJ, 747, 28

Fogarty, K., Postman, M., Connor, T., Donahue, M., \& Moustakas, J. 2015, ApJ, 813, 117

Gaspari, M., Brighenti, F., \& Temi, P. 2012a, MNRAS, 424, 190

Gaspari, M., Ruszkowski, M., \& Sharma, P. 2012b, ApJ, 746, 94

Gilkis, A., \& Soker, N. 2012, MNRAS, 427, 1482

Heinz, S., \& Churazov, E. 2005, ApJ Lett., 634, L141 
Hillel, S., \& Soker, N. 2014, MNRAS, 445, 4161

Hillel, S., \& Soker, N. 2016, MNRAS, 455, 2139

Li, Y., Bryan, G. L., Ruszkowski, M., Voit, G. M., O’Shea, B. W., \& Donahue, M. 2015, ApJ, 811, 73

McCourt, M., Sharma, P., Quataert, E., \& Parrish, I. J. 2012, MNRAS, 419, 3319

McNamara, B. R., Russell, H. R., Nulsen, P. E. J., et al. 2014, ApJ, 785, 44

Mignone, A., Bodo, G., Massaglia, S., et al. 2007, ApJ Suppl. Ser., 170, 228

Moe, M., Arav, N., Bautista, M. A., \& Korista, K. T. 2009, ApJ, 706, 525

Mulchaey, J. S., Davis, D. S., Mushotzky, R. F., \& Burstein, D. 1996, ApJ, 456, 80

Nesvadba, N. P. H., Boulanger, F., Lehnert, M. D., Guillard, P., \& Salome, P. 2011, A\&A, 536, L5

Omma, H., Binney, J., Bryan, G., \& Slyz, A. 2004, MNRAS, 348, 1105

Perucho, M., Martí, J.-M., Quilis, V., \& Ricciardelli, E. 2014, MNRAS, 445, 1462

Pfrommer, C. 2013, ApJ, 779, 10

Pizzolato, F. \& Soker, N. 2005 ApJ, 632, 821

Pizzolato, F., \& Soker, N. 2010, MNRAS, 408, 961

Pope, E. C. D. 2009, MNRAS, 395, 2317

Prasad, D., Sharma, P., \& Babul A. 2015, ApJ, 811, 10

Randall, S. W., Forman, W. R., Giacintucci, S., et al. 2011, ApJ, 726, 86

Randall, S. W., Nulsen, P. E. J., Jones, C., et al. 2015, ApJ, 805, 112

Revaz, Y., Combes, F., \& Salomé, P. 2008, A\&A, 477, L33

Roediger, E., Brüggen, M., Rebusco, P., Böhringer, H., \& Churazov, E. 2007, MNRAS, 375, 15

Russell, H. R., Fabian, A. C., McNamara, B. R., \& Broderick, A. E. 2015, MNRAS, in press

Sharma, P., McCourt, M., Quataert, E., \& Parrish, I. J. 2012, MNRAS, 420, 3174

Soker, N., Akashi, M., Gilkis, A., Hillel, S., Papish, O., Refaelovich, M., \& Tsebrenko, D. 2013, Astronomische Nachrichten, 334, 402

Sternberg, A., Pizzolato, F. \& Soker N. 2007, ApJ, 656, L5 
Sternberg, A., \& Soker N. 2008a, MNRAS, 384, 1327

Sternberg, A., \& Soker, N. 2008b, MNRAS, 389, L13

Sternberg, A., \& Soker, N. 2009, MNRAS, 395, 228

Stevens, I. R., Blondin, J. M., \& Pollock, A. M. T. 1992, ApJ, 386, 265

Sutherland, R. S., \& Dopita, M. A. 1993, ApJ Suppl. Ser., 88, 253

Tremblay, G. R., O'Dea, C. P., Baum, S. A. et al. 2015, MNRAS

Vernaleo, J. C., \& Reynolds, C. S. 2006, ApJ, 645, 83

Voit, G. M., \& Donahue, M. 2015, ApJ Lett., 799, LL1

Voit, G. M., Donahue, M., Bryan, G. L., \& McDonald, M. 2015, Nature, 519, 203

Wagh, B., Sharma, P., \& McCourt, M. 2014, MNRAS, 439, 2822

Walg, S., Achterberg, A., Markoff, S., Keppens, R., \& Meliani, Z. 2013, MNRAS, 433, 1453

Werner, N., Zhuravleva, I., Churazov, E., Simionescu, A., Allen, S. W., Forman, W., Jones, C., \& Kaastra, J. S. 2009, MNRAS, 398, 23

Wilman, R. J., Edge, A. C., McGregor, P. J., \& McNamara, B. R. 2011, MNRAS, 416, 2060

Zhuravleva, I., Churazov, E., Arevalo, P., et al. 2015, MNRAS, 450, 4184

Zhuravleva, I., Churazov, E., Schekochihin, A. A., et al. 2014, Nature, 515, 85 